\title{
Erratum to: Drebrin - From Structure and Function to Physiological and Pathological Roles
}

\author{
Tomoaki Shirao and Yuko Sekino
}

\section{Erratum to:}

T. Shirao, Y. Sekino (eds.), Drebrin, Advances in Experimental Medicine and Biology 1006, DOI 10.1007/978-4-431-56550-5

This book was initially published with error in the title of Part IV, which has now been corrected.

The correct title has been updated in the part page and in the table of contents.

The updated original online version of book can be found at https://doi.org/10.1007/978-4-431-56550-5 